\title{
2009/7
}

Correlated risks, bivariate utility and optimal choices

Michel M. Denuit, Louis Eeckhoudt and Mario Menegatti 
CORE

Voie du Roman Pays 34

B-1348 Louvain-la-Neuve, Belgium.

Tel (32 10) 474304

Fax (32 10) 474301

E-mail: corestat-library@uclouvain.be http://www.uclouvain.be/en-44508.html 


\title{
CORE DISCUSSION PAPER
}

$2009 / 7$

\section{Correlated risks, bivariate utility and optimal choices}

\author{
Michel M. DENUIT ${ }^{1}$, Louis EECKHOUDT ${ }^{2}$ \\ and Mario MENEGATTI ${ }^{3}$
}

February 2009

\begin{abstract}
In this paper, we consider a décision-maker facing a financial risk flanked by a background risk, possibly non-financial, such as health or environmental risk. A decision has to be made about the amount of an investment (in the financial dimension) resulting in a future benefit either in the same dimension (savings) or in the order dimension (environmental quality or health improvement). In the first case, we show that the optimal amount of savings decreases as the pair of risks increases in the bivariate increasing concave dominance rules of higher degrees which express the common preferences of all the decision-makers whose twoargument utility function possesses direct and cross derivatives fulfilling some specific requirements. Roughly speaking, the optimal amount of savings decreases as the two risks become "less positively correlated" or marginally improve in univariate stochastic dominance. In the second case, a similar conclusion on optimal investment is reached under alternative conditions on the derivatives of the utility function.
\end{abstract}

Keywords: bivariate higher order increasing concave stochastic dominance, precautionary savings, background risk, dependence.

\footnotetext{
${ }^{1}$ Université catholique de Louvain, Institut de Sciences Actuarielles \& Institut de Statistique, B-1348 Louvain-la-Neuve, Belgium.

${ }^{2}$ IESEG School of Management, Lille, France; Université catholique de Louvain, CORE, B-1348

Louvain-la-Neuve, Belgium.

${ }^{3}$ Università degli Studi di Parma, Dipartimento di Economia, Parma, Italy.
}

This paper presents research results of the Belgian Program on Interuniversity Poles of Attraction initiated by the Belgian State, Prime Minister's Office, Science Policy Programming. The scientific responsibility is assumed by the authors. 



\section{Introduction and motivation}

In many fields of decision science, the importance of the degree of correlation between the risks faced by private or public decision-makers has been stressed for a long time. The literature on the topic is abundant and a survey would require a paper of its own. However, we start with some examples taken from this literature in order to illustrate the focus of the present paper.

In finance, the early contributions around the capital asset pricing model have illustrated the impact of the covariance between the return of an asset and that of the market for the value of that asset. For example, Sharpe (1970, page 89) already noted that "the reward for bearing risk is [...] equal to some constant times the covariance between the security's rate of return and that for a market as a whole".

In the field of insurance, DoHerTy \& SCHLESInger (1983) have shown that Arrow's famous result about the optimality of the deductible may no longer hold true when the insured faces a background risk that is correlated with the risk to be insured.

Finally, in a recent paper devoted to the impact of background risks for the adoption of new prospects, TSETLIN \& WinkLER (2005) observe that "the optimal decisions in the correlated background risk setting can be very different from the decisions that would be recommended if the correlation were ignored and can be very sensitive to the sign and magnitude of the correlation". Although these papers - as well as many others in the field are written in an expected utility framework, it is worth mentioning that sometimes also a non expected utility approach is adopted. See, e.g., Eichner \& WAGENER $(2003,2008)$.

It is important to notice that for all papers mentioned so far - as well as a vast majority of other ones in the literature - correlated risks are analyzed in the framework of a unidimensional utility function. However, in many real-world circumstances the two possibly correlated risks are expressed in different dimensions. For this reason, a multidimensional utility function was introduced into the analysis of agents' attitude toward risk with reference to the effect of the presence of a background risk on risk aversion. See for instance Kinlstrom \& Mirman (1974), Pratt (1988) and Finkelshtain, Kella \& Scarsini (1999). So far however, a multidimensional risk framework was not used in many problems involving optimal investment decisions. The main purpose of this paper is to fill this gap.

To illustrate the topics discussed in this paper, consider for instance an healthy individual who makes current expenditures (buying expensive diets, regular check-ups, etc.) in order to improve his future health status. When such a decision is made, this individual knows his current wealth and his current health status. However, for the period where the benefits are going to be obtained, there is a joint uncertainty about the level of these variables. Again, we are interested to know how the joint presence of these risks and their possible positive correlation affects the current monetary investment in health.

In this example, the current investment is in one dimension (money) and the future benefit is in the other dimension (health). Notice, however, that this does not need to be the case. For instance, in the standard savings problems, current costs and future benefits are expressed in the same (monetary) dimension and, usually, the utility obtained in each period is assumed to be unidimensional. Here, we extend this case and we consider a decision-maker who has in each period a bidimensional utility (e.g., wealth and health) and we examine how an increased correlation between the future values of these two arguments affects the current 
savings choice.

The results derived in the present paper complement previous studies that were developed mostly in the context of savings decisions. In a paper devoted to the properties of bivariate utility functions, EECKHOUdT, REY \& Schlesinger (2007) examine how current savings are affected when future wealth and health are independent random variables. In a sense, the results they obtain represent a benchmark case for the present paper. Almost simultaneously, Courbage \& REY (2007) analyzed the conditions for the existence of positive precautionary savings in the presence of a non-financial background risk in some specific cases while MENEGATTI (2008a) provided a correct interpretation of some results derived in that paper. Menegatti (2008b) also examined optimal savings in the presence of a small income risk and a small background risk. Relying on a bivariate Taylor expansion, he showed that the existence of precautionary savings depends on two terms capturing the direct effect of income risk and its interaction with background risk.

Our main objectives here are to extend the results of these papers in two directions. First, we will not concentrate exclusively on savings problems in which the costs and benefits of a decision are expressed in a single dimension. Instead, we will also consider problems where two different dimensions of the decision-maker's welfare are affected by his choices. Besides, we do not limit ourselves to small risks but we consider the preferences of decision-makers who dislike an "increase in correlation" between income risk and background risk. Such increases are described by higher degree bivariate stochastic dominance rules, and extend the idea of EPSTEIN \& TANNy (1980) to general risks. This clarifies the conditions leading to an increase in precautionary savings.

The paper is organized as follows. In Section 2, besides introducing notation, we present in a formal manner the problems of choice that have been informally described so far. Then, Section 3 recalls the definition of the $s$-increasing concave stochastic dominance rules, generated by the common preferences of all the decision-makers with a $s$-increasing concave utility function. Section 3 allows for the inclusion of a background risk by considering bivariate utility functions. The univariate $s$-increasing concave dominance rules are extended to dimension 2 by means of the $\left(s_{1}, s_{2}\right)$-increasing concave dominance expressing the common preferences of all the decision-makers with a $\left(s_{1}, s_{2}\right)$-increasing concave utility function. These tools are applied to precautionary savings in Section 4 and to investment in health improvements in Section 5. Finally, Section 6 concludes.

\section{The choice problem}

We consider a decision-maker who has in each of two periods a bivariate utility $u_{t}(\cdot, \cdot), t=$ 0,1 , defined on wealth $x$ and another attribute denoted as $h$ (health, say). Current monetary resources are known with certainty and denoted $x_{0}$. In the current period, the available quantity of the other attribute is also known with certainty and denoted $h_{0}$. Uncertainty prevails about the quantities of each attribute that will be available in the future. These random variables are denoted respectively as $X$ and $H$, with corresponding expectations $\mathbb{E}[X]$ and $\mathbb{E}[H]$.

In this context we examine two kinds of problem. The first one we investigate is a savings problem in which the sacrifice of current consumption increases future consumption 
opportunities. In the second problem, today's expenditures increase the level of the other resource denoted $h$ (i.e. improving future health status)

When we consider the savings problem, the decision-maker's objective is to select the optimal amount of wealth to be transferred from period 0 to period 1 . The choice of saving $a$ is thus made in order to maximize total utility $U$ defined as

$$
U(a)=u_{0}\left(x_{0}-a, h_{0}\right)+\frac{1}{1+\rho} \mathbb{E}\left[u_{1}(a(1+r)+X, H)\right]
$$

where $\rho$ is the subjective discount rate applied to future utility and $r$ is the rate of return on savings. To simplify notation, we assume, without loss of generality, that the intertemporal discount rate and the interest rate are both equal to 0 . The optimal amount of savings $a^{\star}$ is then determined as the solution of the equation

$$
u_{0}^{(1,0)}\left(x_{0}-a, h_{0}\right)=\mathbb{E}\left[u_{1}^{(1,0)}(X+a, H)\right]
$$

where $u_{t}^{\left(k_{1}, k_{2}\right)}$ denotes the $\left(k_{1}, k_{2}\right)$ th cross derivative of $u_{t}$, that is, $u_{t}^{\left(k_{1}, k_{2}\right)}=\frac{\partial^{k_{1}+k_{2}}}{\partial x^{k_{1}} \partial h^{k_{2}}} u_{t}(x, h)$.

With reference to the second problem, we assume that the decision-maker has to choose now how much of resources $x_{0}$ is to be devoted to an investment $(a)$ that will improve his future health by an amount $m \cdot a$ where $m$ represents the productivity of the current monetary sacrifice expressed in units of the other attribute. More precisely, the decisionmaker's objective is to select $a$ in order to maximize $U$ defined by

$$
U(a)=u_{0}\left(x_{0}-a, h_{0}\right)+\frac{1}{1+\rho} \mathbb{E}\left[u_{1}(X, H+m \cdot a)\right] .
$$

To simplify notation, we assume, without loss of generality, that the intertemporal discount rate is equal to 0 and that the productivity $m$ is equal to 1 . The associated first-order condition for a maximum is then given by

$$
u_{0}^{(1,0)}\left(x_{0}-a, h_{0}\right)=\mathbb{E}\left[u_{1}^{(0,1)}(X, H+a)\right] .
$$

As for (2.1), we denote as $a^{\star}$ the solution of (2.2).

In these two problems the optimal choice depends on the decision-maker's attitude both toward intertemporal allocation of wealth and toward risk. With reference to this second aspect, in our framework, the decision-maker faces two risks, related respectively to the uncertainty on $X$ and the one on $H$. This paper will focus on the effect of the correlation between these two risks. Henceforth, we assume that $u_{0}$ is concave in its first argument, i.e. $u_{0}^{(2,0)} \leq 0$. This ensures that the left-hand side of (2.1) and the left-hand side of (2.2) both increase in $a$.

\section{Common preferences of decision-makers with $\left(s_{1}, s_{2}\right)$ - increasing concave utility functions}

In order to introduce bivariate stochastic dominance rules, we first recall some well-known results about the unidimensional case. This also enables us to introduce some notation. 
For $s=1,2, \ldots$, let us define the class $\mathcal{U}_{s-i c v}$ of the regular $s$-increasing concave functions as the class containing all the functions $u$ defined on (a subset of) the real line with derivatives $u^{(1)}, u^{(2)}, \ldots, u^{(s)}$ such that $(-1)^{k+1} u^{(k)} \geq 0$ for $k=1,2, \ldots, s$. The class $\mathcal{U}_{s-i c v}$ thus contains the non-decreasing functions with derivatives of degrees 1 to $s$ with alternating signs. Many commonly used utility functions belong to $\mathcal{U}_{s-i c v}$ for all $s$ (as their derivatives alternate in sign, beginning with positive marginal utility). For instance, all the completely monotone utility functions, including the logarithmic, exponential and power utility functions belong to $\mathcal{U}_{s-i c v}$ for all $s$.

The common preferences of all the decision-makers with $s$-increasing concave utility functions generate the $s$-increasing concave dominance rule, called the $s$-increasing concave order. More precisely, given two random variables $X$ and $Y, X$ is said to be smaller than $Y$ in the $s$-increasing concave order, denoted by $X \preceq_{s-i c v} Y$ when $\mathbb{E}[u(X)] \leq \mathbb{E}[u(Y)]$ for all the functions $u$ in $\mathcal{U}_{s-i c v}$, provided the expectations exist.

The orders $\preceq_{s-i c v}$ are closely related to the increasing sth degree risk of EKERN (1980). For more details, we refer the interested readers to Denuit, LEFÈvRE \& Shaked (1998) and Denuit, De ViJlder \& Lefèvre (1999).

Let us now consider utility functions $u$ defined on (a subset of) the real plane. This allows to account for bidimensional consequences. For instance, one dimension corresponds to monetary variables, income, say, and the other one corresponds to non-monetary variables related to the health status, like the life expectancy for instance.

Recall from Denuit, Lefèvre \& Mesfioui (1999) the definition of the bivariate $\left(s_{1}, s_{2}\right)$-increasing concave order where $s_{1}$ and $s_{2}$ are positive integers. To this end, let us introduce the class $\mathcal{U}_{\left(s_{1}, s_{2}\right)-i c v}$ of the regular $\left(s_{1}, s_{2}\right)$-increasing concave functions defined as the class of all the functions $u$ such that $(-1)^{k_{1}+k_{2}+1} u^{\left(k_{1}, k_{2}\right)} \geq 0$ for all $k_{1}=0,1, \ldots, s_{1}$, $k_{2}=0,1, \ldots, s_{2}$, with $k_{1}+k_{2} \geq 1$. Eeckhoudt, Rey \& Schlesinger (2007) provided equivalence between the signs of the cross-derivatives $u^{\left(k_{1}, k_{2}\right)}$ and individual preferences within a particular class of simple lotteries. This leads for instance to the concepts of cross-prudence and cross-temperance, exhibited by the elements of $\mathcal{U}_{\left(s_{1}, s_{2}\right)-i c v}$ for $s_{1}$ and $s_{2}$ large enough. See also Denuit, Eeckhoudt \& Rey (2008).

Let us consider two bivariate random vectors $\left(X_{1}, X_{2}\right)$ and $\left(Y_{1}, Y_{2}\right)$. Then, $\left(X_{1}, X_{2}\right)$ is said to be smaller than $\left(Y_{1}, Y_{2}\right)$ in the $\left(s_{1}, s_{2}\right)$-increasing concave ordering, denoted by $\left(X_{1}, X_{2}\right) \preceq_{\left(s_{1}, s_{2}\right)-i c v}\left(Y_{1}, Y_{2}\right)$, when $\mathbb{E}\left[u\left(X_{1}, X_{2}\right)\right] \leq \mathbb{E}\left[u\left(Y_{1}, Y_{2}\right)\right]$ for all the functions $u$ in $\mathcal{U}_{\left(s_{1}, s_{2}\right)-i c v}$, provided the expectations exist. We indicate that some special cases of these orderings have been considered before in economics, e.g. in ATKInson \& Bourguignon (1982). We refer to Denuit \& EECKhoudt (2008) for a thorough study of the conditions under which $\preceq_{\left(s_{1}, s_{2}\right)-i c v}$ holds true.

Let us now relate the bivariate $\preceq_{\left(s_{1}, s_{2}\right)-i c v}$ rankings to their univariate counterparts. First, it is easily seen that

$$
\left(X_{1}, X_{2}\right) \preceq_{\left(s_{1}, s_{2}\right)-i c v}\left(Y_{1}, Y_{2}\right) \Rightarrow X_{1} \preceq_{s_{1}-i c v} Y_{1} \text { and } X_{2} \preceq_{s_{2}-i c v} Y_{2}
$$

so that $\preceq_{\left(s_{1}, s_{2}\right)-i c v}$ marginally agrees with $\preceq_{s_{1}-i c v}$ and $\preceq_{s_{2}-i c v}$. The reciprocal implication in (3.1) is true when $X_{1}$ and $X_{2}$ are mutually independent. More precisely, denoting as $\left(X_{1}^{\perp}, X_{2}^{\perp}\right)$ and $\left(Y_{1}^{\perp}, Y_{2}^{\perp}\right)$ two pairs of mutually independent random variables, we have the following equivalence between a joint $\preceq_{\left(s_{1}, s_{2}\right)-i c v}$ ranking and the two marginal $\preceq_{s_{1}-i c v}$ and 
$\preceq_{s_{2}-i c v}$ rankings:

$$
\left(X_{1}^{\perp}, X_{2}^{\perp}\right) \preceq_{\left(s_{1}, s_{2}\right)-i c v}\left(Y_{1}^{\perp}, Y_{2}^{\perp}\right) \Leftrightarrow X_{1}^{\perp} \preceq_{s_{1}-i c v} Y_{1}^{\perp} \text { and } X_{2}^{\perp} \preceq_{s_{2}-i c v} Y_{2}^{\perp} .
$$

Henceforth, we use the superscript " $\perp$ " to emphasize that the corresponding random variables are mutually independent.

Finally, a key feature of the bivariate extension $\preceq_{\left(s_{1}, s_{2}\right)-i c v}$ is that

$$
\left(X_{1}, X_{2}\right) \preceq_{\left(s_{1}, s_{2}\right)-i c v}\left(Y_{1}, Y_{2}\right) \Rightarrow X_{1}+X_{2} \preceq_{\left(s_{1}+s_{2}\right)-i c v} Y_{1}+Y_{2} .
$$

This implication follows from the fact that the bivariate function $\left(x_{1}, x_{2}\right) \mapsto u\left(x_{1}+x_{2}\right)$ belongs to $\mathcal{U}_{\left(s_{1}, s_{2}\right)-i c v}$ when $u$ belongs to $\mathcal{U}_{\left(s_{1}+s_{2}\right)-i c v}$.

\section{Application to savings}

Savings decisions are usually taken in a context where uncertainty affects the future. A first source of uncertainty is related to the variability of future wealth, generating the so-called income risk. However, very often the income risk cannot be considered in isolation, as it is usually flanked by one or more uninsurable background risks (such as health or environmental risks, for instance). Neglecting these background risks can lead to misleading conclusions about decision-maker's optimal choices.

The framework in Section 2 makes it possible to consider how these joint risks affect current savings decisions. In this context, we now address two questions: first, what is the effect of uncertainty on optimal savings in the presence of a background risk and second, how does a deterioration in the higher degree stochastic dominance affect the optimal amount of savings in the 2-period model.

Let us now establish the main result of this section, which will allow us to answer these questions. It is expressed in terms of a $\preceq_{\left(s_{1}, s_{2}\right)-i c v}$ ranking, as this order relation accounts for both marginal changes and modifications in the dependence structure. Specifically, we show that the optimal amount of saving $a^{\star}$ defined as the solution of (2.1) is monotone with respect to $\preceq_{\left(s_{1}, s_{2}\right)-i c v}$ provided the utility function $u_{1}(\cdot, \cdot)$ satisfies some higher degree concavity properties which, as we have seen, have implications for the signs of successive direct and cross derivatives of $u$.

Proposition 4.1. If $u_{1} \in \mathcal{U}_{\left(s_{1}+1, s_{2}\right)-i c v}$ then

$$
\left(X_{1}, H_{1}\right) \preceq_{\left(s_{1}, s_{2}\right)-i c v}\left(X_{2}, H_{2}\right) \Rightarrow a_{1}^{\star} \geq a_{2}^{\star}
$$

where $a_{i}^{\star}, i=1,2$, is the solution of $(2.1)$ with $\left(X_{i}, H_{i}\right)$ substituted for $(X, H)$, respectively.

Proof. To establish that the inequality $a_{1}^{\star} \geq a_{2}^{\star}$ indeed holds, we need to prove that

$$
\mathbb{E}\left[u_{1}^{(1,0)}\left(X_{1}+a, H_{1}\right)\right] \geq \mathbb{E}\left[u_{1}^{(1,0)}\left(X_{2}+a, H_{2}\right)\right]
$$

is valid for any $a \geq 0$. To see this, note that solving (2.1) for $a_{1}^{\star}$ is equivalent to find the intersection point between the non-decreasing curve $a \mapsto u_{0}^{(1,0)}\left(x_{0}-a, h_{0}\right)$ and the lefthand side of (4.1) viewed as a function of $a$. Similarly, solving (2.1) for $a_{2}^{\star}$ requires the 
determination of the intersection point between the same curve and the right-hand side of (4.1) viewed as a function of $a$. Both sides of (4.1) are non-increasing in $a$ because $u$ is concave in its first argument (as $s_{1}+1 \geq 2$ ). If we define the function $v$ as $v=-u_{1}^{(1,0)}$, we clearly see that $v^{\left(k_{1}, k_{2}\right)}=-u_{1}^{\left(k_{1}+1, k_{2}\right)}$. Hence,

$$
u_{1} \in \mathcal{U}_{\left(s_{1}+1, s_{2}\right)-i c v} \Rightarrow v \in \mathcal{U}_{\left(s_{1}, s_{2}\right)-i c v} .
$$

Since we assumed that $\left(X_{1}, H_{1}\right) \preceq_{\left(s_{1}, s_{2}\right)-i c v}\left(X_{2}, H_{2}\right)$ we must have $\mathbb{E}\left[v\left(X_{1}, H_{1}\right)\right] \leq \mathbb{E}\left[v\left(X_{2}, H_{2}\right)\right]$, which shows that the inequality (4.1) is indeed valid. This ends the proof.

This theorem contains as a special case a result derived by EeckHoudT \& Schlesinger (2008) for the case of a univariate utility function.

Now that we are equipped with this general result, let us apply it to different situations. Recall that $a^{\star}$ has been defined as the solution of (2.1). Similarly, define $\bar{a}$ as the solution of $(2.1)$ with $(\mathbb{E}[X], \mathbb{E}[H])$ substituted for $(X, H)$, $\widehat{a}$ as the solution of $(2.1)$ with $(X, \mathbb{E}[H])$ substituted for $(X, H)$, and $\widetilde{a}$ as the solution of $(2.1)$ with $(\mathbb{E}[X], H)$ substituted for $(X, H)$. Henceforth, we compare these different amounts of savings under various assumptions.

The amounts $\bar{a}, \widehat{a}$ and $\widetilde{a}$ can be compared under very general conditions, as they involve a single source of risk for $\widehat{a}$ and $\widetilde{a}$ and no randomness for $\bar{a}$. We then get from Proposition 4.1 that

$$
(X, \mathbb{E}[H]) \preceq_{(2,1)-i c v}(\mathbb{E}[X], \mathbb{E}[H]) \Rightarrow \bar{a} \leq \widehat{a} \text { provided } u_{1} \in \mathcal{U}_{(3,1)-i c v} .
$$

and

$$
(\mathbb{E}[X], H) \preceq_{(1,2)-i c v}(\mathbb{E}[X], \mathbb{E}[H]) \Rightarrow \bar{a} \leq \widetilde{a} \text { provided } u_{1} \in \mathcal{U}_{(2,2)-i c v}
$$

Let us now investigate the standard case of an income risk $X$ flanked by an independent background risk $H$. Then, by (3.2) we have

$$
(X, H) \preceq_{(1,2)-i c v}(X, \mathbb{E}[H]) \Rightarrow \widehat{a} \leq a^{\star} \text { provided } u_{1} \in \mathcal{U}_{(2,2)-i c v} .
$$

and

$$
(X, H) \preceq_{(2,1)-i c v}(\mathbb{E}[X], H) \Rightarrow \widetilde{a} \leq a^{\star} \text { provided } u_{1} \in \mathcal{U}_{(3,1)-i c v}
$$

Finally, using together (4.2) and (4.4) we get

$$
\bar{a} \leq a^{\star} \text { provided } u_{1} \in \mathcal{U}_{(3,2)-i c v} .
$$

The same inequality is obtained from (4.3) and (4.5).

Some of the previous comparisons have a clear economic interpretation. First, when the inequality $\widetilde{a} \leq a^{\star}$ holds, the amount of optimal savings under uncertainty is larger compared to the situation where future income is known with certainty. This positive extrasaving $a^{\star}-\widetilde{a}$ is called positive "precautionary saving". Second, when the inequality $\bar{a} \leq a^{\star}$ holds, the amount of optimal savings increases compared to the situation where both future income and future health status (environmental quality) are both known with certainty. This positive extra-saving $a^{\star}-\bar{a}$ is called "two-source precautionary saving" by MENEGATTI (2008b). Finally, the comparison between $a^{\star}$ and $\widetilde{a}$ identifies the partial effect of uncertainty on savings due to background risk. Indeed, when the inequality $\widehat{a} \leq a^{\star}$ holds, the case where the amount of optimal savings increases compared to the situation where future health status 
(environmental quality) is known with certainty. We can call this positive extra-saving $a^{\star}-\widehat{a}$ the "precautionary saving due to background risk".

The effect of the marginals on the optimal savings is also clear from Proposition 4.1 in the independent case. Specifically, consider independent random variables $X_{1}^{\perp}, X_{2}^{\perp}, H_{1}^{\perp}$, and $H_{2}^{\perp}$. By (3.2), we easily get

$$
\left.\begin{array}{l}
X_{1}^{\perp} \preceq_{s_{1}-i c v} X_{2}^{\perp} \\
H_{1}^{\perp} \preceq_{s_{2}-i c v} H_{2}^{\perp}
\end{array}\right\} \Rightarrow a_{1}^{\star} \geq a_{2}^{\star} \text { provided } u_{1} \in \mathcal{U}_{\left(s_{1}+1, s_{2}\right)-i c v} .
$$

Hence, a deterioration of the income risk and/or of the background risk in a higher degree concave sense leads the decision-maker to increase optimal savings when $u_{1} \in \mathcal{U}_{\left(s_{1}+1, s_{2}\right)-i c v}$, which means that the direct and cross derivatives of $u_{1}$ exhibit the appropriate sequence of signs.

Now that the effect of letting some components (income or background risk) become random is well understood as long as they remain independent, let us allow for some kinds of dependence between $X$ and $H$. Indeed, in general, it seems reasonable to believe that when the background risk $H$ is positively related to the income risk $X$, every source of uncertainty increases saving, so that we have a positive "precautionary saving", a positive "precautionary saving due to background risk" and a positive "two-source precautionary saving".

Let us start the analysis of this point with the following simple and intuitive example.

Example 4.2. Assume that both $X_{i}$ and $H_{i}, i=1,2$, only assume two values, $x_{1}$ and $x_{2}$ for $X_{i}$, with $x_{1}<x_{2}$, and $h_{1}$ and $h_{2}$ for $H_{i}$, with $h_{1}<h_{2}$, say. The marginal distributions of $X_{i}$ and $H_{i}$ are given by $\operatorname{Pr}\left[X_{i}=x_{1}\right]=1-\operatorname{Pr}\left[X_{i}=x_{2}\right]=p_{X}$ and $\operatorname{Pr}\left[H_{i}=h_{1}\right]=$ $1-\operatorname{Pr}\left[H_{i}=h_{2}\right]=p_{H}$. Hence, both $\left(X_{1}, H_{1}\right)$ and $\left(X_{2}, H_{2}\right)$ have the same univariate marginals. Without real loss of generality, we assume that $p_{X} \leq p_{H}$. Let us now consider $\rho$ such that $-p_{X} p_{H} \leq \rho \leq p_{X}\left(1-p_{H}\right)$ and define the joint distribution of $\left(X_{i}, H_{i}\right)$ as

$$
\begin{aligned}
& \operatorname{Pr}\left[X_{i}=x_{1}, H_{i}=h_{1}\right]=p_{X} p_{H}+\rho_{i} \\
& \operatorname{Pr}\left[X_{i}=x_{2}, H_{i}=h_{1}\right]=\left(1-p_{X}\right) p_{H}-\rho_{i} \\
& \operatorname{Pr}\left[X_{i}=x_{1}, H_{i}=h_{2}\right]=p_{X}\left(1-p_{H}\right)-\rho_{i} \\
& \operatorname{Pr}\left[X_{i}=x_{2}, H_{i}=h_{2}\right]=\left(1-p_{X}\right)\left(1-p_{H}\right)+\rho_{i} .
\end{aligned}
$$

When the dependence parameter $\rho$ increases, we face a correlation increasing transformation as defined by EPSTEIN \& TANNY (1980) and a correlation averse decision-maker should then dislike an increase in $\rho$. It is easily seen that

$$
\rho_{2} \leq \rho_{1} \Rightarrow\left(X_{1}, H_{1}\right) \preceq_{(1,1)-i c v}\left(X_{2}, H_{2}\right) \Rightarrow a_{1}^{\star} \geq a_{2}^{\star}
$$

provided $u_{1} \in \mathcal{U}_{(2,1)-i c v}$ by virtue of Proposition 4.1. Thus, we deduce that the optimal amount of savings increases with the dependence parameter $\rho$ in this case.

This simple model, thus, confirms our intuition: increasing the positive dependence between $X$ and $H$ (in the $\preceq_{(1,1)-i c v}$-sense) increases the optimal savings as soon as $u_{1}$ is $(2,1)$ increasing concave, i.e. when the derivatives of $u_{1}$ satisfy $u^{(1,1)} \leq 0, u^{(2,0)} \leq 0, u^{(1,0)} \geq 0$, $u^{(0,1)} \geq 0$ and $u^{(2,1)} \geq 0$. 
Given this simple example, let us now examine some more general kinds of dependence between $X$ and $H$. First, recall that $(X, H)$ is said to be positively quadrant dependent (PQD, in short) if the inequality $\operatorname{Pr}[X>x, H>h] \geq \operatorname{Pr}[X>x] \operatorname{Pr}[H>h]$ holds for all $x$ and $h$ or, equivalently, if the inequality $\operatorname{Pr}[X \leq x, H \leq h] \geq \operatorname{Pr}[X \leq x] \operatorname{Pr}[H \leq h]$ holds for all $x$ and $h$. We see from this definition that when $(X, H)$ is PQD, its components $X$ and $H$ are more likely to be large together (or to be small together) compared with the theoretical situation in which $X$ and $H$ are independent. Similarly, $(X, H)$ is said to be negatively quadrant dependent (NQD, in short) if the inequality $\operatorname{Pr}[X>x, H>h] \leq$ $\operatorname{Pr}[X>x] \operatorname{Pr}[H>h]$ holds for all $x$ and $h$ or, equivalently, if the inequality $\operatorname{Pr}[X \leq x, H \leq$ $h] \leq \operatorname{Pr}[X \leq x] \operatorname{Pr}[H \leq h]$ holds for all $x$ and $h$.

These dependence notions can be defined with the help of $\preceq_{(1,1)-i c v}$. To this end, let us define the random couple $\left(X^{\perp}, H^{\perp}\right)$ with mutually independent components and such that $X^{\perp}$ and $X$ are identically distributed, as well as $H^{\perp}$ and $H$. Then, we know from Denuit \& Eeckhoud $(2008)$ that $(X, H) \mathrm{PQD} \Leftrightarrow(X, H) \preceq_{(1,1)-i c v}\left(X^{\perp}, H^{\perp}\right)$ and $(X, H) \mathrm{NQD} \Leftrightarrow$ $\left(X^{\perp}, H^{\perp}\right) \preceq_{(1,1)-i c v}(X, H)$. Denoting as $a^{\perp}$ the solution of (2.1) with $\left(X^{\perp}, H^{\perp}\right)$ substituted for $(X, H)$, we then have the following result which directly follows from Proposition 4.1.

Property 4.3. Assume that $u_{1} \in \mathcal{U}_{(2,1)-i c v}$. Then,

(i) $(X, H) P Q D \Rightarrow a^{\star} \geq a^{\perp}$;

(ii) $(X, H) N Q D \Rightarrow a^{\star} \leq a^{\perp}$.

Positive dependence (formalized by PQD) increases the optimal amount of savings compared to independence, whereas negative dependence (formalized here by NQD) decreases it provided $u_{1} \in \mathcal{U}_{(2,1)-i c v}$, that is, provided the partial derivatives of $u_{1}$ exhibit the appropriate sequence of signs.

Another case to be analysed is the hypothesis of a positive correlation between the two risks which can be formalised by assuming that $\mathbb{E}[X \mid H]$ is non-decreasing in $H$. This means that the income decreases on average when health deteriorates. This ensures for instance that $X$ and $H$ are positively correlated as $\operatorname{Cov}[X, H]=\mathbb{C o v}[\mathbb{E}[X \mid H], H] \geq 0$ since the covariance between two non-decreasing functions of the same random variable $H$ is always non-negative.

Given this assumption, the next result states sufficient conditions to have a positive precautionary saving and to have a positive two-source precautionary saving.

Property 4.4. Assume that $\mathbb{E}[X \mid H]$ is non-decreasing in $H$. Then

(i) $u_{1} \in \mathcal{U}_{(3,1)-i c v} \Rightarrow a^{\star} \geq \widetilde{a}$;

(ii) $u_{1} \in \mathcal{U}_{(3,2)-i c v} \Rightarrow a^{\star} \geq \bar{a}$.

Proof. ¿From Proposition 4.1, we have to show that $(X, H) \preceq_{(2,1)-i c v}(\mathbb{E}[X], H)$ holds true to establish the validity of (i). Consider $v \in \mathcal{U}_{(2,1)-i c v}$. As a first step, note that since $v$ is concave in its first argument, we have

$$
\mathbb{E}[v(X+a, H)]=\mathbb{E}[\mathbb{E}[v(X+a, H) \mid H]] \leq \mathbb{E}[v(\mathbb{E}[X \mid H]+a, H)]
$$


As $\mathbb{E}[X \mid H]$ and $H$ are comonotonic by assumption, we know that $(\mathbb{E}[X \mid H], H)$ is the least

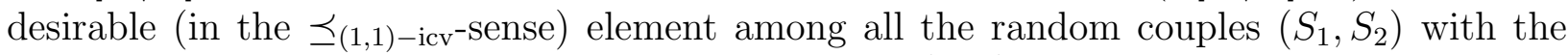
same marginal distributions. Taking in particular $\left(S_{1}^{\perp}, S_{2}^{\perp}\right)$ with independent components, we thus have

$$
(\mathbb{E}[X \mid H], H) \preceq_{(1,1)-\mathrm{icv}}\left(S_{1}^{\perp}, S_{2}^{\perp}\right) .
$$

This simply follows from the fact that the joint distributions function of $(\mathbb{E}[X \mid H], H)$ dominates all those of $\left(S_{1}, S_{2}\right)$ by virtue of the Fréchet-Höffding inequality. Now, we have from (3.2) that

$$
\left(S_{1}^{\perp}, S_{2}^{\perp}\right) \preceq(2,1)-\mathrm{icv}\left(\mathbb{E}\left[S_{1}\right], S_{2}^{\perp}\right) .
$$

Note that $\mathbb{E}\left[S_{1}\right]=\mathbb{E}[\mathbb{E}[X \mid H]]=\mathbb{E}[X]$. Hence, we get by transitivity that $(X, H) \preceq(2,1)$-icv $(\mathbb{E}[X], H)$, which ends the proof of (i).

Let us now turn to (ii). As $\mathcal{U}_{(3,2)-i c v} \subset \mathcal{U}_{(3,1)-i c v}$, we know that $a^{\star} \geq \widetilde{a}$ by (i). Furthermore, as $\mathcal{U}_{(3,2)-i c v} \subset \mathcal{U}_{(2,2)-i c v}$, we know from (4.3) that $\widetilde{a} \geq \bar{a}$. The announced result then follows by combining these two inequalities.

It would be tempting to conclude that the opposite results hold true in case of negative dependence between $X$ and $H$, if $\mathbb{E}[X \mid H]$ is non-increasing in $H$, say. However, matters are much more complicated in this case, as there is an adverse effect of letting some components become random but a beneficial effect due to negative correlation (so that the background risk hedges the income risk). The question then becomes which effect dominates. These conclusions are coherent with those obtained by MENEGATTI (2008b) in the case of small risks. In this case Menegatti (2008b) derives the conditions describing the comparison between $a^{\star}$ and $\widetilde{a}$ and between $a^{\star}$ and $\bar{a}$. These two conditions are given by

$$
a^{\star} \geq \widetilde{a} \Leftrightarrow \operatorname{Var}[X] u^{(3,0)}+2 \mathbb{C o v}[X, H] u^{(2,1)} \geq 0
$$

and

$$
a^{\star} \geq \bar{a} \Leftrightarrow \operatorname{Var}[X] u^{(3,0)}+2 \mathbb{C o v}[X, H] u^{(2,1)}+\operatorname{Var}[X] u^{(1,2)} \geq 0
$$

It is easy to see that, given the conditions on the utility function in Property 4.4, a positive correlation between $X$ and $H$ ensures that these two inequalities are satisfied while a negative correlation generates ambiguous results. In many applications, however, a non-negative correlation is expected between $X$ and $H$ (think about health and income, for instance) so that the case of negative dependence is less relevant for the problems treated in the present paper.

Let us now perform the same analysis for the background risk $H$. Specifically, we investigate whether there is a positive precautionary saving efect, that is, whether the optimal amount of savings increases when $X$ and $H$ are positively related compared to the situation where $H$ is known with certainty. The existence of a positive two-source precautionary saving is also studied.

Property 4.5. Assume that $\mathbb{E}[H \mid X]$ is non-decreasing in $X$. Then

(i) $u_{1} \in \mathcal{U}_{(2,2)-i c v} \Rightarrow a^{\star} \geq \widehat{a}$;

(ii) $u_{1} \in \mathcal{U}_{(3,2)-i c v} \Rightarrow a^{\star} \geq \bar{a}$. 
Proof. Result (i) holds true if we can prove that $(X, H) \preceq_{(1,2)-i c v}(X, \mathbb{E}[H])$. This can be established proceeding as in Property 4.4(i). Specifically, taking $v \in \mathcal{U}_{(1,2)-i c v}$ we have

$$
\mathbb{E}[v(X, H)] \leq \mathbb{E}[v(X, \mathbb{E}[H \mid X])]
$$

by Jensen inequality. Now, since $(X, \mathbb{E}[H \mid X])$ is comonotonic and denoting as $\left(S_{1}^{\perp}, S_{2}^{\perp}\right)$ a random couple with the same marginal distributions as $(X, \mathbb{E}[H \mid X])$, we have

$$
(X, \mathbb{E}[H \mid X]) \preceq_{(1,1)-i c v}\left(S_{1}^{\perp}, S_{2}^{\perp}\right) \preceq_{(1,2)-i c v}\left(S_{1}^{\perp}, \mathbb{E}\left[S_{2}^{\perp}\right]\right)
$$

so that

$$
\mathbb{E}[v(X, \mathbb{E}[H \mid X])] \leq \mathbb{E}\left[v\left(S_{1}^{\perp}, \mathbb{E}\left[S_{2}^{\perp}\right]\right)\right]=\mathbb{E}[v(X, \mathbb{E}[H])]
$$

whence (i) follows. Now, considering (ii), the result follows from the inclusions $\mathcal{U}_{(3,2)-i c v} \subset$ $\mathcal{U}_{(2,2)-i c v}$, so that (i) applies and $\mathcal{U}_{(3,2)-i c v} \subset \mathcal{U}_{(3,1)-i c v}$, so that (4.3) holds true.

There are many cases in which the assumptions behind Properties 4.4-4.5 hold true. For instance, this will be the case if $\operatorname{Pr}[X>x \mid H=h]$ is non-decreasing in $h$ for every $x$, and if $\operatorname{Pr}[H>h \mid X=x]$ is non-decreasing in $x$ for every $h$, a situation referred to in the literature as conditional increasingness. Many well-known copula families enjoy this property, so that the results derived in this section apply when the dependence structure of $(X, H)$ is described by such copulas. We refer the reader to Chapters 4-6 in DenUiT ET AL. (2005) for more details.

\section{Application to health investments}

The second kind of problem described in Section 2 concerns a current investment improving future environmental quality or health status. As an illustration, we consider here that the second argument of the utility function is health quality.

As in the case of the savings problem, this decision is usually taken in the presence of different sources of uncertainty pertaining either to future resources ("income risk", described by the random variable $X$ ) or to future health quality ("non-financial background risk", described by the random variable $H$ ). Both these different sources of uncertainty affect the optimal choice of the decision-maker. As in in Section 4, we can identify some plausible consequences of the features of correlation between the two risks on optimal investment. In this respect, there are essentially two concerns:

- there is risk both on the value of the future resources and on future health status. A common intuition suggests that risk on health should induce a risk-averse decisionmaker to increase his current investment in health improvement while we have less intuition about the impact of the risk on future resources.

- it is very likely that the two risks are positively correlated since a deterioration in the health status is usually accompanied by a lower income.

Let us first derive the main result of this section, which parallels Proposition 4.1. 
Proposition 5.1. If $u_{1} \in \mathcal{U}_{\left(s_{1}, s_{2}+1\right)-i c v}$ then

$$
\left(X_{1}, H_{1}\right) \preceq_{\left(s_{1}, s_{2}\right)-i c v}\left(X_{2}, H_{1}\right) \Rightarrow a_{1}^{\star} \geq a_{2}^{\star}
$$

where $a_{i}^{\star}, i=1,2$, is the solution of $(2.2)$ with $\left(X_{i}, H_{i}\right)$ substituted for $(X, H)$, respectively.

Proof. The proof is analogous to that of Property 4.1. Specifically, defining $v=-u_{1}^{(0,1)}$ we have that $v^{\left(k_{1}, k_{2}\right)}=-u_{1}^{\left(k_{1}, k_{2}+1\right)}$ so that $u_{1} \in \mathcal{U}_{\left(s_{1}, s_{2}+1\right)-i c v} \Rightarrow v \in \mathcal{U}_{\left(s_{1}, s_{2}\right)-i c v}$. Hence,

$$
\begin{aligned}
& \left(X_{1}, H_{1}\right) \preceq_{\left(s_{1}, s_{2}\right)-i c v}\left(X_{2}, H_{2}\right) \Rightarrow \mathbb{E}\left[v\left(X_{1}, H_{1}\right)\right] \leq \mathbb{E}\left[v\left(X_{2}, H_{2}\right)\right] \\
& \Rightarrow \mathbb{E}\left[u_{1}^{(0,1)}\left(X_{1}, H_{1}\right)\right] \geq \mathbb{E}\left[u_{1}^{(0,1)}\left(X_{2}, H_{2}\right)\right] \\
& \Rightarrow a_{1}^{\star} \geq a_{2}^{\star}
\end{aligned}
$$

which ends the proof.

As in the previous section, define $a^{\star}$ as the solution of (2.2), $\bar{a}$ as the solution of (2.2) with $(\mathbb{E}[X], \mathbb{E}[H])$ substituted for $(X, H), \widehat{a}$ as the solution of $(2.2)$ with $(X, \mathbb{E}[H])$ substituted for $(X, H)$, and $\widetilde{a}$ as the solution of $(2.2)$ with $(\mathbb{E}[X], H)$ substituted for $(X, H)$. We then get from Proposition 5.1 that

$$
(\mathbb{E}[X], H) \preceq(1,2)-i c v(\mathbb{E}[X], \mathbb{E}[H]) \Rightarrow \bar{a} \leq \widetilde{a} \text { provided } u_{1} \in \mathcal{U}_{(1,3)-i c v}
$$

and

$$
(X, \mathbb{E}[H]) \preceq_{(2,1)-i c v}(\mathbb{E}[X], \mathbb{E}[H]) \Rightarrow \bar{a} \leq \widehat{a} \text { provided } u_{1} \in \mathcal{U}_{(2,2)-i c v} .
$$

It is interesting to compare (4.2)-(4.3) to (5.2)-(5.1).

Let us now investigate the standard case of an income risk $X$ flanked by an independent background risk $H$. Then, by (3.2) we have

$$
(X, H) \preceq_{(2,1)-i c v}(\mathbb{E}[X], H) \Rightarrow \widetilde{a} \leq a^{\star} \text { provided } u_{1} \in \mathcal{U}_{(2,2)-i c v}
$$

and

$$
(X, H) \preceq_{(1,2)-i c v}(X, \mathbb{E}[H]) \Rightarrow \widehat{a} \leq a^{\star} \text { provided } u_{1} \in \mathcal{U}_{(1,3)-i c v} .
$$

Finally, using together inequalities (5.1) and (5.3), or alternatively inequalities (5.2) together with (5.4), we get

$$
\bar{a} \leq a^{\star} \text { provided } u_{1} \in \mathcal{U}_{(2,3)-i c v} .
$$

The interpretation of these results is similar to the interpretation given in Section 4 . When the inequality $\widetilde{a} \leq a^{\star}$ holds, the amount of optimal investment under uncertainty is larger compared to the situation where future income is known with certainty, generating a positive extra-investment in health due to income risk. When the inequality $\widehat{a} \leq a^{\star}$ holds, the amount of optimal investment increases compared to the situation where future health status is known with certainty, generating a positive extra-investment in health due to health risk. Finally, when the inequality $\bar{a} \leq a^{\star}$ holds, the amount of optimal investment increases compared to the situation where both future income and future health status are known with certainty, generating a positive extra-investment in health due to the contemporaneous presence of the two risks. 
The effect of the marginals on the optimal investments is also clear in the independent case, that is, when the income $X_{i}^{\perp}$ is independent of the background risk $H_{i}^{\perp}, i=1,2$. From (3.2), we easily get

$$
\left.\begin{array}{l}
X_{1}^{\perp} \preceq_{s_{1}-i c v} X_{2}^{\perp} \\
H_{1}^{\perp} \preceq_{s_{2}-i c v} H_{2}^{\perp}
\end{array}\right\} \Rightarrow a_{1}^{\star} \geq a_{2}^{\star} \text { provided } u_{1} \in \mathcal{U}_{\left(s_{1}, s_{2}+1\right)-i c v} .
$$

Let us now consider the impact of dependence on optimal investments. As in the application to saving, it seems plausible to to believe that when the background risk $H$ is positively related to the income risk $X$, every source of uncertainty increases optimal investment. The following analysis examines this problem.

Coming back to Example 4.2, we can provide a first intuitive result.

Example 5.2 (Example $4.2 \mathrm{Ctd}$ ). Taking $u_{1} \in \mathcal{U}_{(1,2)-i c v}$ we see that

$$
\rho_{2} \leq \rho_{1} \Rightarrow\left(X_{1}, H_{1}\right) \preceq_{(1,1)-i c v}\left(X_{2}, H_{2}\right) \Rightarrow a_{1}^{\star} \geq a_{2}^{\star}
$$

so that optimal investments increase with the dependence parameter $\rho$ in this simple model.

The impact of switching from mutually independent $X$ and $H$ to PQD or NQD ones is also clear, as shown in the next result which follows from Proposition 5.1 exactly as Property 4.3 was deduced from Proposition 4.1.

Property 5.3. Assume that $u_{1} \in \mathcal{U}_{(1,2)-i c v}$. Then,

(i) $(X, H) P Q D \Rightarrow a^{\star} \geq a^{\perp}$;

(ii) $(X, H) N Q D \Rightarrow a^{\star} \leq a^{\perp}$.

Finally we can examine the case of positive correlation formalized by the increasingness of $X$ in $H$, or of $H$ in $X$, on average. Proceeding as we did for Properties 4.4 and 4.5, we get the following results.

Property 5.4. Assume that $\mathbb{E}[H \mid X]$ is non-decreasing in $X$. Then,

(i) $u_{1} \in \mathcal{U}_{(1,3)-i c v} \Rightarrow a^{\star} \geq \widehat{a}$;

(ii) $u_{1} \in \mathcal{U}_{(2,3)-i c v} \Rightarrow a^{\star} \geq \bar{a}$.

Proof. To establish (i), we need to prove that $(X, H) \preceq_{(1,2)-i c v}(X, \mathbb{E}[H])$, which has been shown to hold in the proof of Property 4.5. Result (ii) then follows from the inclusions $\mathcal{U}_{(2,3)-i c v} \subset \mathcal{U}_{(1,3)-i c v}$ and $\mathcal{U}_{(2,3)-i c v} \subset \mathcal{U}_{(2,2)-i c v}$ so that by (i) and (5.2) we have $a^{\star} \geq \widehat{a} \geq \bar{a}$.

Property 5.5. Assume that $\mathbb{E}[X \mid H]$ is non-decreasing in $H$. Then

(i) $u_{1} \in \mathcal{U}_{(2,2)-i c v} \Rightarrow a^{\star} \geq \widetilde{a}$;

(ii) $u_{1} \in \mathcal{U}_{(2,3)-i c v} \Rightarrow a^{\star} \geq \bar{a}$. 
Proof. Item (i) follows from the stochastic inequality $(X, H) \preceq(2,1)-i c v(\mathbb{E}[X], H)$, which has been shown to be valid in the proof of Property 4.4. Then, (ii) is deduced from the inclusions $\mathcal{U}_{(2,3)-i c v} \subset \mathcal{U}_{(2,2)-i c v}$ and $\mathcal{U}_{(2,3)-i c v} \subset \mathcal{U}_{(1,3)-i c v}$ so that by (i) together with (5.1) we get $a^{\star} \geq \widetilde{a} \geq \bar{a}$.

Note that the conditions on function $u_{1}$ in Property 5.4(i) and Proposition 5.1, requiring respectively $u_{1} \in \mathcal{U}_{(1,3)-i c v}$ and $u_{1} \in \mathcal{U}_{\left(s_{1}, s_{2}+1\right)-i c v}$, correspond to conditions $u_{1} \in \mathcal{U}_{(3,1)-i c v}$ and $u_{1} \in \mathcal{U}_{\left(s_{1}+1, s_{2}\right)-i c v}$ in Property 4.4(i) and Proposition 4.1. Finally, considering these conditions together, it is easy to see that if $u_{1} \in \mathcal{U}_{(3,3)-i c v}$ then the conditions for all the results in Properties 4.4, 4.5, 5.4 and 5.5 are satisfied. The same occurs with reference to Propositions 4.1 and 5.1 if $u_{1} \in \mathcal{U}_{\left(s_{1}+1, s_{2}+1\right)-i c v}$.

\section{Conclusion}

The impact of background risks on optimal decisions has been an intensive topic of research for the last twenty years. So far, the debate took place mostly under the assumption that the decision-maker has a univariate objective which forces the two risks to be expressed in the same dimension.

The purpose of this paper was to extend the discussion to the (realistic) case of background risks in a multidimensional setting, with a special emphasis on the impact of a positive correlation between these risks. While some nuances appear when the current effort and the future results are either in the same or in a different dimension, basically some regularity conditions on the alternating signs of successive direct and cross derivatives of the bidimensional utility function lead to results in line with the basic intuition that a positive correlation between income and background risks induces more effort today to face them. As a result, the basic message that was already present in the early contributions on background risks and precautionary motives by Kimball (1990) and EeckHoudt \& Kimball (1992) is reinforced and substantially extended.

\section{Acknowledgements}

Michel Denuit acknowledges the financial support of the Communauté française de Belgique under contract "Projet d'Actions de Recherche Concertées" ARC 04/09-320, as well as the financial support of the Banque Nationale de Belgique under grant "Risk measures and Economic capital".

\section{References}

Atkinson, A.B., and Bourguignon, F. (1982). The comparison of multi-dimensioned distributions of economic status. Review of Economic Studies 49, 183-201.

Courbage, C., \& Rey, B. (2007). Precautionary saving in the presence of other risks. Economic Theory 32, 417-424. 
Denuit, M., De Vijlder, F.E., \& Lefèvre, Cl. (1999). Extremal generators and extremal distributions for the continuous s-convex stochastic orderings. Insurance: Mathematics and Economics 24, 201-217.

Denuit, M., Dhaene, J., Goovaerts, M.J., \& Kaas, R. (2005). Actuarial Theory for Dependent Risks: Measures, Orders and Models. Wiley, New York.

Denuit, M., \& Eeckhoudt, L. (2008). Bivariate stochastic dominance and common preferences of decision-makers with risk independent utilities. Working Paper 08-03, Institut des Sciences Actuarielles, Université Catholique de Louvain, Louvain-la-Neuve, Belgium.

Denuit, M., Eeckhoudt, L., \& Rey, B. (2008). Some consequences of correlation aversion in decision science. Annals of Operations Research, in press.

Denuit, M., Lefèvre, Cl., \& Mesfioui, M. (1999). A class of bivariate stochastic orderings with applications in actuarial sciences. Insurance: Mathematics and Economics 24, 31-50.

Denuit, M., Lefèvre, Cl., \& Shaked, M. (1998). The s-convex orders among real random variables, with applications. Mathematical Inequalities and Their Applications 1, 585613.

Doherty, N.A., \& Schlesinger H. (1983). Optimal insurance in incomplete markets. Journal of Political Economy 91, 1045-1054.

Eeckhoudt, L., \& Kimball, M.S. (1992). Background risk, prudence and the demand for insurance. In "Contributions to Insurance Economics", edited by G. Dionne, Kluwer Academic Publishers, pp. 239-254.

Eeckhoudt, L., Rey, B., \& Schlesinger, H. (2007). A good sign for multivariate risk taking. Management Science 53, 117-124

Eeckhoudt, L., \& Schlesinger, H. (2008). Changes in risk and the demand for savings. Journal of Monetary Economics 55, 1329-1336.

Eichner, T., \& Wagener, A. (2003). Variance vulnerability, background risks and meanvariance preferences. The Geneva Papers on Risk and Insurance - Theory 28, 173-184.

Eichner, T., \& Wagener, A. (2008). Multiple risks and mean-variance preferences. Discussion Paper.

Ekern, S. (1980). Increasing $n$th degree risk. Economics Letters 6, 329-333.

Epstein, L.G., \& Tanny, S.M. (1980). Increasing generalized correlation: A definition and some economic consequences. Canadian Journal of Economics 13, 16-34.

Finkelshtain, I., Kella, O., \& Scarsini, M. (1999). On risk aversion with two risks. Journal of Mathematical Economics 31, 239-250.

Kihlstrom, R.E., \& Mirman, L.J. (1974). Risk aversion with many commodities. Journal of Economic Theory 8, 361-388.

Kimball, M.S. (1990). Precautionary savings in the small and in the large. Econometrica $58,53-73$.

Menegatti, M. (2008a). Precautionary saving in the presence of other risks: A comment. Economic Theory, in press. 
Menegatti, M. (2008b). Optimal saving in the presence of two risks. Journal of Economics, in press

Pratt, J.W. (1988). Aversion to one risk in the presence of others. Journal of Risk and Uncertainty 1, 395-413.

Sharpe, W.F. (1970). Portfolio Theory and Capital Markets. McGraw-Hill Series in Finance.

Tsetlin, I., \& Winkler, R. (2005). Risky choices and correlated background risk. Management Science 51, 1336-1345. 


\section{Recent titles}

\section{CORE Discussion Papers}

2008/53. Roland Iwan LUTTENS and Marie-Anne VALFORT. Voting for redistribution under desertsensitive altruism.

2008/54. Sergei PEKARSKI. Budget deficits and inflation feedback. 2008/55. Raouf BOUCEKKINE, Jacek B. KRAWCZYK and Thomas VALLEE. Towards an understanding of tradeoffs between regional wealth, tightness of a common environmental constraint and the sharing rules.

2008/56. Santanu S. DEY. A note on the split rank of intersection cuts.

2008/57. Yu. NESTEROV. Primal-dual interior-point methods with asymmetric barriers.

2008/58. Marie-Louise LEROUX, Pierre PESTIEAU and Gregory PONTHIERE. Should we subsidize longevity?

2008/59. J. Roderick McCRORIE. The role of Skorokhod space in the development of the econometric analysis of time series.

2008/60. Yu. NESTEROV. Barrier subgradient method.

2008/61. Thierry BRECHET, Johan EYCKMANS, François GERARD, Philippe MARBAIX, Henry TULKENS and Jean-Pascal VAN YPERSELE. The impact of the unilateral EU commitment on the stability of international climate agreements.

2008/62. Giorgia OGGIONI and Yves SMEERS. Average power contracts can mitigate carbon leakage.

2008/63. Jean-Sébastien TANCREZ, Philippe CHEVALIER and Pierre SEMAL. A tight bound on the throughput of queueing networks with blocking.

2008/64. Nicolas GILLIS and François GLINEUR. Nonnegative factorization and the maximum edge biclique problem.

2008/65. Geir B. ASHEIM, Claude D'ASPREMONT and Kuntal BANERJEE. Generalized timeinvariant overtaking.

2008/66. Jean-François CAULIER, Ana MAULEON and Vincent VANNETELBOSCH. Contractually stable networks.

2008/67. Jean J. GABSZEWICZ, Filomena GARCIA, Joana PAIS and Joana RESENDE. On Gale and Shapley 'College admissions and stability of marriage'.

2008/68. Axel GAUTIER and Anne YVRANDE-BILLON. Contract renewal as an incentive device. An application to the French urban public transport sector.

2008/69. Yuri YATSENKO and Natali HRITONENKO. Discrete-continuous analysis of optimal equipment replacement.

2008/70. Michel JOURNÉE, Yurii NESTEROV, Peter RICHTÁRIK and Rodolphe SEPULCHRE. Generalized power method for sparse principal component analysis.

2008/71. Toshihiro OKUBO and Pierre M. PICARD. Firms' location under taste and demand heterogeneity.

2008/72. Iwan MEIER and Jeroen V.K. ROMBOUTS. Style rotation and performance persistence of mutual funds.

2008/73. Shin-Huei WANG and Christian M. HAFNER. Estimating autocorrelations in the presence of deterministic trends.

2008/74. Yuri YATSENKO and Natali HRITONENKO. Technological breakthroughs and asset replacement.

2008/75. Julio DÁVILA. The taxation of capital returns in overlapping generations economies without financial assets.

2008/76. Giorgia OGGIONI and Yves SMEERS. Equilibrium models for the carbon leakage problem.

2008/77. Jean-François MERTENS and Anna RUBINCHIK. Intergenerational equity and the discount rate for cost-benefit analysis.

2008/78. Claire DUJARDIN and Florence GOFFETTE-NAGOT. Does public housing occupancy increase unemployment?

2008/79. Sandra PONCET, Walter STEINGRESS and Hylke VANDENBUSSCHE. Financial constraints in China: firm-level evidence. 


\section{Recent titles}

\section{CORE Discussion Papers - continued}

2008/80. Jean GABSZEWICZ, Salome GVETADZE, Didier LAUSSEL and Patrice PIERETTI. Pubic goods' attractiveness and migrations.

2008/81. Karen CRABBE and Hylke VANDENBUSSCHE. Are your firm's taxes set in Warsaw? Spatial tax competition in Europe.

2008/82. Jean-Sébastien TANCREZ, Benoît ROLAND, Jean-Philippe CORDIER and Fouad RIANE. How stochasticity and emergencies disrupt the surgical schedule.

2008/83. Peter RICHTÁRIK. Approximate level method.

2008/84. Çağatay KAYI and Eve RAMAEKERS. Characterizations of Pareto-efficient, fair, and strategyproof allocation rules in queueing problems.

2009/1. Carlo ROSA. Forecasting the direction of policy rate changes: The importance of ECB words.

2009/2. Sébastien LAURENT, Jeroen V.K. ROMBOUTS and Francesco VIOLANTE. Consistent ranking of multivariate volatility models.

2009/3. Dunia LÓPEZ-PINTADO and Juan D. MORENO-TERNERO. The principal's dilemma.

2009/4. Jacek B. KRAWCZYK and Oana-Silvia SEREA. A viability theory approach to a two-stage optimal control problem of technology adoption.

2009/5. Jean-François MERTENS and Anna RUBINCHIK. Regularity and stability of equilibria in an overlapping generations model with exogenous growth.

2009/6. Nicolas GILLIS and François GLINEUR. Using underapproximations for sparse nonnegative matrix factorization.

2009/7. Michel M. DENUIT, Louis EECKHOUDT and Mario MENEGATTI. Correlated risks, bivariate utility and optimal choices.

\section{Books}

H. TULKENS (ed.) (2006), Public goods, environmental externalities and fiscal competition. New York, Springer-Verlag.

V. GINSBURGH and D. THROSBY (eds.) (2006), Handbook of the economics of art and culture. Amsterdam, Elsevier.

J. GABSZEWICZ (ed.) (2006), La différenciation des produits. Paris, La découverte.

L. BAUWENS, W. POHLMEIER and D. VEREDAS (eds.) (2008), High frequency financial econometrics: recent developments. Heidelberg, Physica-Verlag.

P. VAN HENTENRYCKE and L. WOLSEY (eds.) (2007), Integration of AI and OR techniques in constraint programming for combinatorial optimization problems. Berlin, Springer.

P-P. COMBES, Th. MAYER and J-F. THISSE (eds.) (2008), Economic geography: the integration of regions and nations. Princeton, Princeton University Press.

J. HINDRIKS (ed.) (2008), Au-delà de Copernic: de la confusion au consensus ? Brussels, Academic and Scientific Publishers.

\section{CORE Lecture Series}

C. GOURIÉROUX and A. MONFORT (1995), Simulation Based Econometric Methods.

A. RUBINSTEIN (1996), Lectures on Modeling Bounded Rationality.

J. RENEGAR (1999), A Mathematical View of Interior-Point Methods in Convex Optimization.

B.D. BERNHEIM and M.D. WHINSTON (1999), Anticompetitive Exclusion and Foreclosure Through Vertical Agreements.

D. BIENSTOCK (2001), Potential function methods for approximately solving linear programming problems: theory and practice.

R. AMIR (2002), Supermodularity and complementarity in economics.

R. WEISMANTEL (2006), Lectures on mixed nonlinear programming. 